\title{
Giant orbital moments of Fe and Co in alkali metals $\mathrm{Cs}, \mathrm{Rb}$, and $\mathrm{K}$
}

\author{
G. Y. Guo* \\ Department of Physics, National Taiwan University, Taipei, Taiwan 106, Republic of China \\ and Synchrotron Radiation Research Center, Hsinchu, Taiwan 300, Republic of China
}

(Received 23 December 1999)

\begin{abstract}
Orbital-polarization corrected relativistic spin-density-functional calculations of $\mathrm{Fe}$ and Co in alkali metals $(\mathrm{K}, \mathrm{Rb}$, and $\mathrm{Cs})$ predict that unlike other transition-metal systems where the orbital magnetic moments are quenched, $\mathrm{Fe}$ and $\mathrm{Co}$ in alkali metals possess a giant orbital moment of 2-3 $\mu_{B}$ along with a large spin moment. The induced moments on the host atoms are small. The large orbital moments result from the weak interaction between the $3 d$ impurities and the host atoms. The results reveal the origin of the giant moments observed in $\mathrm{Fe}$ and $\mathrm{Co}$ in $\mathrm{Cs}$ films.
\end{abstract}

Recently, it was reported ${ }^{1}$ that $\mathrm{Fe}$ and $\mathrm{Co}$ impurities on the surface and in the interior of thin Cs films have a large magnetic moment of about 7 and $8 \mu_{B}$, respectively. This interesting result was found in anomolous Hall effect experiments. Since bulk $\mathrm{Fe}$ and $\mathrm{Co}$ have a magnetic moment of only 2.2 and $1.7 \mu_{B},{ }^{2}$ respectively, the observed moments were justifiably considered to be giant. ${ }^{1}$ There is a wellknown system with giant moments: $3 d$ transition-metal impurities in a palladium host possess a large moment $\left(9-12 \mu_{B}\right.$ for $\mathrm{Fe}$ and $\mathrm{Co}$ in $\left.\mathrm{Pd}\right){ }^{3,4}$ For transition-metal compounds, this perhaps had been the only example that have giant moments. The reason is that the Fermi level in bulk Pd is located at the upper edge of the $4 d$ band with a large density of states on it. As a result, Pd is a highly enhanced paramagnet. The presence of a $3 d$ magnetic impurity induces significant spin moments of the order of $0.1 \mu_{B}$ on the neighboring $\mathrm{Pd}$ atoms even including those $\mathrm{Pd}$ atoms being many atomic shells away. ${ }^{3-5}$ Consequently, the total magnetic moment of the $3 d$ impurity plus the surrounding $\mathrm{Pd}$ atoms is gigantic, with the majority being made up of the induced spin moments on the $\mathrm{Pd}$ atoms. However, alkali metals are a good example of nearly-free-electron systems, as described in many solid-state text books. ${ }^{6}$ Indeed, the Fermi surface of $\mathrm{Na}, \mathrm{K}$, and $\mathrm{Rb}$ determined by de Haas-van Alphen experiments deviates from that of the free-electron model by $1 \%$ at worst. $^{6}$ Thus, alkali metals are a weak paramagnet at best and the giant moments in Cs films would not be expected to have the same origin as that of the palladium systems. The presence of the giant moments of $\mathrm{Fe}$ and $\mathrm{Co}$ in $\mathrm{Cs}$ films is therefore a mystery.

In order to find the origin of the observed giant moments of $\mathrm{Fe}$ and $\mathrm{Co}$ in $\mathrm{Cs}$ films, we have calculated from firstprinciples the magnetic moments and other properties of $\mathrm{Fe}$ and $\mathrm{Co}$ in some alkali metals $(\mathrm{K}, \mathrm{Rb}$, and $\mathrm{Cs}$ ) and also of $\mathrm{Fe}$ in transition metals $\mathrm{Ag}$ and $\mathrm{Pd}$. The results of these calculations predict as expected that the induced magnetic moments on the host alkali atoms are small (within $0.02 \mu_{B} /$ atom). However, we find that $\mathrm{Fe}(\mathrm{Co})$ in the alkali metals possesses a giant orbital moment of $2.3 \mu_{B}\left(3.0 \mu_{B}\right)$ as well as a large spin moment of $3.9 \mu_{B}\left(2.7 \mu_{B}\right)$. This finding is surprising at first glance because in transition-metal solids the orbital moment of the $d$ valence electrons is generally quenched due to the crystal-field spitting or the hybridization of the $d$ orbitals with that of neighboring atoms. ${ }^{6}$ For example, bulk Fe has an orbital moment of only $0.09 \mu_{B}$ and bulk Co, $0.14 \mu_{B} \cdot{ }^{2}$ Even in ultrathin $\mathrm{Co} / \mathrm{Au}$ multilayers in which the orbital magnetization is enhanced, the orbital moments of Co atoms are a small fraction (about $0.2 \mu_{B}$ ) of $3.0 \mu_{B} \cdot{ }^{7}$ The results of the present calculations are thus interesting and important. First of all, this theoretical finding sheds light on the mystery of $\mathrm{Fe}$ and $\mathrm{Co}$ in $\mathrm{Cs}$ films ${ }^{1}$ mentioned above. The calculated total angular moment is 3.9 for $\mathrm{Fe}$ in $\mathrm{Cs}$ and 4.1 for $\mathrm{Co}$ in $\mathrm{Cs}$, in good agreement with the experimental values (3.5 and 4.0). ${ }^{1}$ The larger total magnetic moments reported in Ref. 1 could be attributed to the use of Lande factor $g$ of 2 which is appropriate if the orbital moments were negligible. ${ }^{6}$ Second, if the prediction confirmed by experiments, e.g., X-ray magnetic circular dichroism, ${ }^{2} \mathrm{Fe}$ and $\mathrm{Co}$ in alkali metals would be a rare transition-metal system with giant orbital moments and could provide a valuable system for exploring orbital magnetism and for technological applications. Orbital magnetization is known to be closely related to such interesting phenomena as magneto-optical effect, ${ }^{8}$ magnetostriction and magnetocrystalline anisotropy. 7,9

The magnetic moments in Fe (Co) in bcc Cs were determined by performing all-electron self-consistent spinpolarized electronic band-structure calculations. For comparison, the same calculations have also been carried out for $\mathrm{Fe}$ in bcc $\mathrm{K}(\mathrm{Rb})$ and in fcc $\mathrm{Ag}(\mathrm{Pd})$. The well established linear muffin-tin orbital (LMTO) method $^{10}$ has been used. These calculations are based on the first-principles densityfunctional theory with the local spin-density approximation (LDA). ${ }^{11}$ The accurate local exchange-correlation potential parametrized by Vosko et al. ${ }^{13}$ was used. The basis functions for all the atoms used were $s, p$, and $d$ MTO's. The Fe (Co) atoms in the alkali metals were assumed to be substitutional impurities and were modeled by a $3 \times 3 \times 3$ bcc supercell with one $\mathrm{Fe}(\mathrm{Co})$ atom and $53 \mathrm{Cs}(\mathrm{K}, \mathrm{Rb})$ atoms per unit cell. The Fe impurities in $\mathrm{Ag}(\mathrm{Pd})$ were modeled by a $2 \times 2 \times 2$ fcc supercell with one $\mathrm{Fe}$ atom and $31 \mathrm{Ag}(\mathrm{Pd})$ atoms per unit cell. As will be shown below, the induced moments on the near-neighbor (NN) host atoms in all the systems except Fe in Pd are small, and hence the calculated magnetic moments would not be affected if the supercell size were increased. Indeed, the magnetic moments of $\mathrm{Fe}(\mathrm{Co})$ in the alkali metals calculated using a $2 \times 2 \times 2$ supercell (16 atoms/cell) are 
close to that obtained by using a $3 \times 3 \times 3$ supercell. For example, the calculated $\mathrm{Fe}(\mathrm{Co})$ spin and orbital moments in the two cases differ by only $2 \%$. The atomic-sphere radii used were determined from the measured unit-cell volumes of pure $\mathrm{Fe}, \mathrm{Co}, \mathrm{K}, \mathrm{Rb}, \mathrm{Cs}, \mathrm{Ag}$, and Pd metals. ${ }^{6}$ Thus, the lattice constants of the supercells for $\mathrm{Fe}(\mathrm{Co})$ in $\mathrm{Cs}(\mathrm{K}, \mathrm{Rb})$ are about three times that of bulk $\mathrm{Cs}(\mathrm{K}, \mathrm{Rb})$, respectively, and those for $\mathrm{Fe}$ in $\mathrm{Ag}(\mathrm{Pd})$ are about twice that of bulk $\mathrm{Ag}$ $(\mathrm{Pd})$. Note that there could be structural relaxations around the $\mathrm{Fe}(\mathrm{Co})$ atoms in $\mathrm{Cs}$ since the $\mathrm{NN} \mathrm{Fe}(\mathrm{Co})$-Cs distance in the assumed structure is considerably larger than that of the usual $\mathrm{Fe}(\mathrm{Co})$-transition-metal atoms, e.g., in $\mathrm{Fe}$ in $\mathrm{Ag}$. While this possible reduction of the NN Fe-Cs distance must await further experiments (e.g., extended X-ray absorption fine structure), test calculations for $\mathrm{Fe}$ in $\mathrm{Cs}$ with a reduced lattice constant indicated that a reduction of the $\mathrm{Fe}(\mathrm{Co})-\mathrm{Cs}$ distance by up to $22 \%$ would not change significantly the calculated spin and orbital moments of $\mathrm{Fe}(\mathrm{Co})$ in $\mathrm{Cs}$ presented below. The number of $k$ points in the irreducible Brillouin zone (BZ) wedge used in the tetrahedron BZ integration $^{14}$ is 35 for all the systems. Test calculations showed that with these choices of the number of $k$ points, the calculated moments converge to within $5 \%$. For the density of states calculations, $455 k$ points is used for all the systems.

The orbital moments in a transition-metal system result from the spin-orbit splitting of the valence $d$ states. Thus, to calculate the orbital moments one has to either include the spin-orbit coupling as a perturbation in the Kohn-Sham equation ${ }^{10}$ or solve the Kohn-Sham-Dirac equation based on relativistic spin-density-functional theory ${ }^{15}$ (RLDA). We choose the latter and use a fully relativistic extension of the LMTO method. ${ }^{12}$ It is well known that although (R)LDA calculations describe well the spin moments in many solids, they often give too small orbital moments. For example, for bulk $\mathrm{Fe}$ and $\mathrm{Co}$, the theoretical values account for only about $50 \%$ of the measured ones. ${ }^{2}$ A solution to this defect is to include in the Kohn-Sham equation an orbital-polarization correction $^{16}$ (OPC) of $-B L_{z}^{\sigma}$ where $B$ is the Racah parameter (for $d$ states) and $L_{z}^{\sigma}$ the total orbital momentum for spin $\sigma$. Since both $B$ and $L_{z}^{\sigma}$ can be recalculated in every selfconsistent iteration, this scheme is parameter free. The OPC has been found to bring the calculated orbital moments in many metallic solids in good agreement with experiments. ${ }^{16-18}$ In this paper, we have therefore carried out the RLDA calculations with the OPC included through a recent implementation for the Kohn-Sham-Dirac equation. ${ }^{18}$ Because of the time-reversal symmetry breaking and hence the tripling of the irreducible BZ wedge, the number of $k$ points used in the RLDA calculations are three times that used in the LDA calculations.

The calculated magnetic moments for $\mathrm{Fe}$ in $\mathrm{K}, \mathrm{Rb}$, and $\mathrm{Cs}$ are summarized in Table I, and that for $\mathrm{Co}$ in $\mathrm{Cs}$ and $\mathrm{Fe}$ in $\mathrm{Ag}$ and $\mathrm{Pd}$ in Table II. The results of the LDA, RLDA, and RLDA plus OPC calculations are all listed for comparison. Tables I and II show that including OPC typically increases the calculated orbital moments by $30-90 \%$. Since this increase in the orbital moments due to OPC is important to diminish the discrepancy between the LDA calculations and the experiments, ${ }^{2,17,18}$ we will discuss below only the orbital moments obtained by the OPC calculations. The induced orbital moments on the alkali and Ag atoms are all less than
TABLE I. Calculated spin $\left(m_{s}\right)$, orbital $\left(m_{o}\right)$ and total $\left(m_{t}\right.$ $=m_{s}+m_{o}$ ) magnetic moments as well as magnetic dipole moment $\left(T_{z}\right)$ of $\mathrm{Fe}$ in bulk $\mathrm{K}, \mathrm{Rb}$, and $\mathrm{Cs}$. The results of local spin-density approximation (LDA), relativistic LDA (RLDA), and RLDA plus the orbital polarization correction (OPC) calculations are all listed for comparison. $n_{d}\left(n_{t}\right)$ denotes the number of $d$ (valence) electrons of Fe. $M_{t}\left(M_{o}\right)$ denotes the total (orbital) moment per supercell and the superscript $n$ denotes the Cs atoms on the $n$th neighbor atomic shell.

\begin{tabular}{|c|c|c|c|c|c|}
\hline System & Atom & & $\operatorname{LDA}\left(\mu_{B}\right)$ & $\operatorname{RLDA}\left(\mu_{B}\right)$ & OPC $\left(\mu_{B}\right)$ \\
\hline \multirow[t]{5}{*}{$\mathrm{Fe}$ in $\mathrm{K}$} & \multirow[t]{5}{*}{$\mathrm{Fe}$} & $m_{s}$ & 3.75 & 3.75 & 3.68 \\
\hline & & $m_{o}\left(n_{d}\right)$ & & $1.26(6.39)$ & $2.39(6.45)$ \\
\hline & & $m_{t}\left(n_{t}\right)$ & 3.75 & $5.01(7.43)$ & $6.07(7.46)$ \\
\hline & & $7 T_{z}$ & & & -1.25 \\
\hline & & $M_{t}\left(M_{o}\right)$ & 3.52 & $4.65(1.24)$ & $5.76(2.35)$ \\
\hline \multirow[t]{5}{*}{$\mathrm{Fe}$ in $\mathrm{Rb}$} & \multirow[t]{5}{*}{$\mathrm{Fe}$} & $m_{s}$ & 3.86 & 3.86 & 3.79 \\
\hline & & $m_{o}\left(n_{d}\right)$ & & $1.35(6.30)$ & $2.32(6.37)$ \\
\hline & & $m_{t}\left(n_{t}\right)$ & 3.86 & $5.21(7.48)$ & $6.11(7.50)$ \\
\hline & & $7 T_{z}$ & & & -1.38 \\
\hline & & $M_{t}\left(M_{o}\right)$ & 3.46 & $4.69(1.33)$ & $5.61(2.30)$ \\
\hline \multirow[t]{7}{*}{$\mathrm{Fe}$ in $\mathrm{Cs}$} & \multirow[t]{4}{*}{$\mathrm{Fe}$} & $m_{s}$ & 3.86 & 3.86 & 3.86 \\
\hline & & $m_{o}\left(n_{d}\right)$ & & $1.35(6.24)$ & $2.27(6.31)$ \\
\hline & & $m_{t}$ & 3.86 & $5.21(7.53)$ & $6.13(7.55)$ \\
\hline & & $7 T_{z}$ & & & -1.48 \\
\hline & $\mathrm{Cs}^{1,2}$ & $m_{s}$ & -0.02 & -0.02 & -0.02 \\
\hline & \multirow[t]{2}{*}{$\mathrm{Cs}^{3,4}$} & $m_{s}$ & -0.01 & -0.01 & -0.01 \\
\hline & & $M_{t}\left(M_{o}\right)$ & 3.46 & $4.69(1.36)$ & $5.45(2.25)$ \\
\hline
\end{tabular}

$0.01 \mu_{B}$ and are thus not listed in Tables I and II. Table I shows that the calculated spin moment of an iron (cobalt) atom in bulk Cs is 3.9 (2.7) $\mu_{B}$, being short of 4 (3) $\mu_{B}$ predicted by Hund's first rule for a free $d^{6}\left(d^{7}\right)$ ion. ${ }^{6}$ This spin moment reduces slightly as $\mathrm{Cs}$ is replaced by lighter alkali metals $(\mathrm{Rb}, \mathrm{K})$. It is stated in many solid-state textbooks (e.g., Ref. 6) that in $3 d$ transition-metal compounds, the weak spin-orbit coupling of the valence $d$ electrons is suppressed by the crystal fields because of the strong interaction of the $d$ orbitals with neighboring atoms. Thus, Hund's second rule proposed for free ions is no longer applicable and the orbital moment of the valence electrons in these systems is quenched. ${ }^{6}$ A good measure of the crystalfield strength is the energy separation of the $d-t_{2 g}$ and $d$ $-e_{g}$ of the cubic metals at the BZ center. Our calculations show that for bulk Fe, this separation is about $1.6 \mathrm{eV}$, being much larger than the spin-orbit splitting of $0.04 \mathrm{eV}$. It is thus surprising at first glance that the theoretical orbital moment of an iron or colbalt atom in Cs is gigantic $\left(2.3\right.$ or $\left.3.0 \mu_{B}\right)$ (Tables I and II), being 20 times larger that of bulk Fe or Co. As expected, the induced magnetic moments on the host alkali metal atoms in the $\mathrm{NN}$ shell are small (about $\left.-0.02 \mu_{B}\right)$. Furthermore, these small induced moments are antiparallel to that of the iron atoms. The induced magnetic moments on the alkali metal atoms in the third NN shell and beyond are negligible. The total magnetic moment of the $\mathrm{Fe}$ (Co) in Cs system thus consists mostly of the magnetic moment on the $\mathrm{Fe}(\mathrm{Co})$ atom.

The spin moments in an Fe impurity in $\mathrm{Ag}$ are similar to that of Fe in alkali metals, viz., the Fe impurity has a spin moment much larger than that of bulk $\mathrm{Fe}$ and the induced 
TABLE II. Calculated spin $\left(m_{s}\right)$, orbital $\left(m_{o}\right)$ and total $\left(m_{t}\right)$ magnetic moments as well as magnetic dipole moment $\left(T_{z}\right)$ in Co in bulk $\mathrm{Cs}$ and $\mathrm{Fe}$ in bulk $\mathrm{Ag}$ and $\mathrm{Pd}$. The results of local spindensity approximation (LDA), relativistic LDA (RLDA), and RLDA plus the orbital polarization correction (OPC) are all listed for comparison. $n_{d}\left(n_{t}\right)$ denotes the number of $d$ (valence) electrons of Fe. $M_{t}\left(M_{o}\right)$ denotes the total (orbital) moment per supercell and the superscript $n$ denotes the host atoms on the $n$th neighbor atomic shell.

\begin{tabular}{|c|c|c|c|c|c|}
\hline System & Atom & & $\operatorname{LDA}\left(\mu_{B}\right)$ & $\operatorname{RLDA}\left(\mu_{B}\right)$ & $\mathrm{OPC}\left(\mu_{B}\right)$ \\
\hline \multirow[t]{5}{*}{ Co in $\mathrm{Cs}$} & \multirow[t]{5}{*}{ Co } & $m_{s}$ & 2.67 & 2.66 & 2.67 \\
\hline & & $m_{o}\left(n_{d}\right)$ & & $2.24(7.43)$ & $2.95(7.45)$ \\
\hline & & $m_{t}\left(n_{t}\right)$ & 2.67 & $4.90(8.61)$ & $5.62(8.64)$ \\
\hline & & $7 T_{z}$ & & & 0.23 \\
\hline & & $M_{t}\left(M_{o}\right)$ & 2.11 & $4.38(2.23)$ & $5.21(2.94)$ \\
\hline \multirow[t]{6}{*}{$\mathrm{Fe}$ in $\mathrm{Ag}$} & \multirow[t]{4}{*}{$\mathrm{Fe}$} & $m_{s}$ & 3.08 & 3.08 & 3.07 \\
\hline & & $m_{o}\left(n_{d}\right)$ & & $0.22(6.52)$ & $0.68(6.52)$ \\
\hline & & $m_{t}\left(n_{t}\right)$ & 3.08 & $3.30(7.51)$ & $3.75(7.51)$ \\
\hline & & $7 T_{z}$ & & & -0.13 \\
\hline & \multirow[t]{2}{*}{$\mathrm{Ag}^{1}$} & $m_{s}$ & -0.01 & -0.01 & -0.01 \\
\hline & & $M_{t}$ & 2.98 & 3.07 & 3.52 \\
\hline \multirow[t]{6}{*}{$\mathrm{Fe}$ in $\mathrm{Pd}$} & \multirow[t]{4}{*}{$\mathrm{Fe}$} & $m_{s}$ & 3.36 & 3.35 & 3.34 \\
\hline & & $m_{o}\left(n_{d}\right)$ & & $0.07(6.41)$ & $0.22(6.41)$ \\
\hline & & $m_{t}\left(n_{t}\right)$ & 3.36 & $3.42(7.62)$ & $3.56(7.62)$ \\
\hline & & $7 T_{z}$ & & & -0.003 \\
\hline & \multirow[t]{2}{*}{$\mathrm{Pd}^{1}$} & $m_{s}$ & 0.28 & 0.26 & 0.28 \\
\hline & & $m_{o}$ & & 0.027 & 0.028 \\
\hline
\end{tabular}

spin moments on the neighboring $\mathrm{Ag}$ atoms are negligibly small (see Tables I and II). On the other hand, the orbital moment $\left(0.68 \mu_{B}\right)$ of Fe impurity in bulk Ag is small compared with that of the alkali metal systems though it is enhanced relative to that of bulk Fe. This results from a stronger interaction between the $\mathrm{Fe} d$ orbitals and the host $\mathrm{Ag}$ bands. To see this clearly, we display the LDA density of states (DOS) spectra of the Fe impurities in Cs, $\mathrm{Ag}$, and $\mathrm{Pd}$ in Fig. 1. Figure 1 shows that spin-up $d$ band, spin-up and down $s$ bands of $\mathrm{Fe}$ in $\mathrm{Cs}$ are located below the Cs conduction band and thus do not hybridize with Cs $s p$ orbitals. As a result, they are highly localized and their DOS are a sharp peak located, respectively, at 3.2, 2.7, and $2.0 \mathrm{eV}$ below the Fermi level $\left(E_{f}\right)$ [see Fig. 1(a)]. Though in overlap with the Cs $s p$ bands, Fe spin-down $d$ band forms a narrow resonant peak with an estimated width at half-height of $0.05 \mathrm{eV}$, due to weak interaction with $\mathrm{Cs} s p$ bands. Note that the small $\mathrm{Fe}$ DOS component present in the Cs band region [Fig. 1(a)] is due to $\mathrm{Fe} s$ orbital. In constrast, spin-up and down $d$ bands of $\mathrm{Fe}$ in Ag are much broader [Fig. 1(b)]. Further, a small component of the Fe $d$ DOS extends to the entire $\mathrm{Ag} d$ band region. This stronger interaction between the $\mathrm{Fe}$ and $\mathrm{Ag} d$ electrons is also seen in the size of the Fe $3 d$ crystal-field spitting in $\mathrm{Fe}$ in $\mathrm{Ag}$ which is about $0.10 \mathrm{eV}$ (spin-down band) compared with $0.01 \mathrm{eV}$ (spin-down band) for $\mathrm{Fe}$ in $\mathrm{Cs}$. The spin moment of the Fe impurity in $\mathrm{Pd}$ is in the same order of magnitude as that of $\mathrm{Fe}$ in $\mathrm{Ag}$ and alkali metals (see Tables I and II). However, because of the very strong interaction between the $d$ electrons of $\mathrm{Fe}$ and $\mathrm{Pd}$ atoms as revealed in the almost complete mixing of the $\mathrm{Fe}$ and $\mathrm{Pd}$ spin-up DOS in the entire valence band [see Fig. 1(c)], the
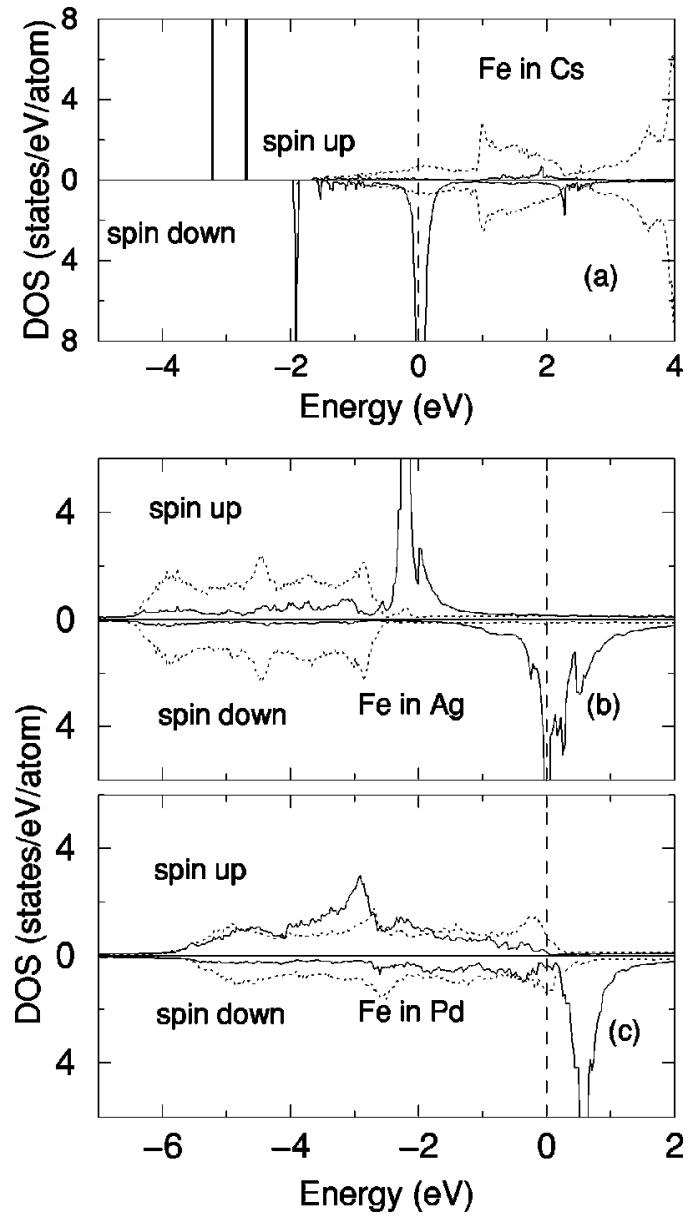

FIG. 1. Density of states (DOS) of Fe in Cs (a), Ag (b), and Pd (c). Solid curves are for the Fe atom. Dotted curves in (a), (b), and (c) denote the averaged DOS for the $\mathrm{Cs}, \mathrm{Ag}$, and $\mathrm{Pd}$ atoms, respectively. The Fermi level is at the zero energy.

induced moments on the near-neighbor host Pd atoms are considerable (Table II) and extended to several nextneighboring atomic shells. ${ }^{5}$ On the other hand, the very strong interaction between the $d$ electrons of $\mathrm{Fe}$ and Pd atoms in the Pd system quenches the orbital moment of the $\mathrm{Fe}$ atoms $\left(0.2 \mu_{B}\right)$. The $\mathrm{Fe} 3 d$ crystal-field splitting defined above in this system is about $0.27 \mathrm{eV}$, being much larger that in $\mathrm{Fe}$ in $\mathrm{Cs}$ and also larger than the spin-orbit splitting (0.04 $\mathrm{eV}$ ).

The reported total magnetic moment is $7 \mu_{B}$ for Fe in Cs and $8 \mu_{B}$ for Co in Cs. ${ }^{1}$ The experimental values appear to be larger than the corresponding theoretical values of 5.5 and $5.2 \mu_{B}$. However, this direct comparison should be made with caution. In Ref. 1 , the total angular momentum $(J=L$ $+S)$ of the systems was derived by fitting a Brillouin function ${ }^{6}$ to the measured anomalous Hall resistances (AHR) with assumptions that the AHR is proportional to the magnetization and that the Lande factor $g(J L S)=2$. The total angular momentum $(J)$ was found to be 3.5 for $\mathrm{Fe}$ in $\mathrm{Cs}$ and 4.0 for $\mathrm{Co}$ in $\mathrm{Cs}$, in reasonable agreement with the corresponding theoretical values of 3.9 and 4.1. The total magnetic moments were obtained by using expression $M_{t}$ $=g J \mu_{B} \cdot{ }^{1}$ The assumption that $g=2$ is correct if the orbital angular momentum $L=0$. The assumption was obviously reasonable since no transition-metal system had been known 
to possess a significant orbital moment before. For Fe and Co in alkali metals, however, the present calculations show that the orbital moments are huge. As a result, the $g$ estimated using the calculated orbital and spin moments is significantly reduced. This $g$ value is about 1.42 (1.28) for $\mathrm{Fe}(\mathrm{Co})$ in $\mathrm{Cs}$, being close to $\frac{3}{2}\left(\frac{4}{3}\right)$ for a free $\mathrm{Fe}^{2+}\left(\mathrm{Co}^{2+}\right)$ ion. ${ }^{6}$ If these $g$ factors were used, the total magnetic moments would have been 5.0 for $\mathrm{Fe}$ in $\mathrm{Cs}$ and $5.1 \mu_{B}$ for $\mathrm{Co}$ in $\mathrm{Cs}$, being close to the theoretical results. This would suggest that another analysis of the AHR data ${ }^{1}$ might be needed. On the other hand, other magnetic experiments will be useful. In particular, $\mathrm{x}$-ray magnetic circular dichroism (XMCD) has recently emerged as an element-specific probe of spin and orbital magnetic moments in solids. ${ }^{19,20,2}$ The prediction of the giant orbital moments of $\mathrm{Fe}$ and $\mathrm{Co}$ in alkali metals could be checked by a soft x-ray $\mathrm{Fe}(\mathrm{Co}) L_{2,3}$-edge MCD measurement. Furthermore, the negligibly small induced moments on the host alkali atoms could be verified by measuring e.g., $\mathrm{K}$ $K$-edge MCD spectrum. It should be pointed out that unlike other transition-metal intermetallics where the magnetic dipole moments are small, ${ }^{20,21}$ the magnetic dipole moment $\left(T_{z}\right)$ (Ref. 22) on the Fe impurity in alkali metals is large (see Table I) and cannot be neglected when deriving the spin

*Electronic address: gyguo@phys.ntu.edu.tw

${ }^{1}$ H. Beckmann and G. Bergmann, Phys. Rev. Lett. 83, 2417 (1999).

${ }^{2}$ C.T. Chen et al., Phys. Rev. Lett. 75, 152 (1995).

${ }^{3}$ G.J. Nienwenhuys, Adv. Phys. 24, 515 (1975).

${ }^{4}$ R.P. Peters et al., Phys. Rev. Lett. 53, 1108 (1984).

${ }^{5}$ R. Zeller, Modell. Simul. Mater. Sci. Eng. 1, 553 (1993).

${ }^{6}$ E.g., N.W. Ashcroft and N.D. Mermin, Solid State Physics (Saunders College, Philadelphia, 1976).

${ }^{7}$ D. Weller et al., Phys. Rev. Lett. 75, 3752 (1995).

${ }^{8}$ D. Weller et al. Phys. Rev. Lett. 72, 2097 (1994).

${ }^{9}$ H.A. Dürr et al., Science 277, 213 (1997).

${ }^{10}$ O.K. Andersen, Phys. Rev. B 12, 3060 (1975).

${ }^{11}$ P. Hohenberg and W. Kohn, Phys. Rev. 136, B864 (1964); W. Kohn and L.J. Sham, Phys. Rev. 140, A1133 (1965). moment from the XMCD measurements via the spin sum rule. $^{20,2}$

Summarizing, in order to reveal the origin of the recently observed giant moments of $\mathrm{Fe}$ and $\mathrm{Co}$ in $\mathrm{Cs}$ films, we have carried orbital-polarization-corrected relativistic spin-density function calculations for $\mathrm{Fe}$ and $\mathrm{Co}$ in some alkali metals (K, $\mathrm{Rb}$, and $\mathrm{Cs}$ ). These calculations predict that $\mathrm{Fe}$ and $\mathrm{Co}$ in the alkali metals possess a giant orbital magnetic moment. Since RLDA-OPC calculations have been found to give accurate orbital moments, $\mathrm{Fe}$ and $\mathrm{Co}$ in the alkali metals are expected to be the first transition-metal systems with giant orbital moments. The predicted giant orbital moments together with the large spin moments on the Fe and Co impurities are believed to be the origin of the giant moments observed in $\mathrm{Fe}$ and $\mathrm{Co}$ in Cs films. It is hoped that this interesting finding would prompt further experimental investigations into these systems, in particular, direct magnetization measurements such as X-ray magnetic circular dichroism.

The author thanks C.T. Chen, M.-C. Chang, and G. Bergmann for stimulating discussions, and the National Science Council of ROC for financial support (NSC 88-2112-M0020043, NSC 89-2112-M002-0025).

${ }^{12}$ H. Ebert, Phys. Rev. 38, 9391 (1988).

${ }^{13}$ S.H. Vosko, L. Wilk, and M. Nusair, Can. J. Phys. 58, 1200 (1980).

${ }^{14}$ O. Jepsen and O.K. Andersen, Solid State Commun. 9, 1763 (1971).

${ }^{15}$ A.K. Rajagopal, J. Phys. C 11, L943 (1978); A.H. MacDonald and S.H. Vosko, ibid. 17, 3355 (1979).

${ }^{16}$ M.S.S. Brooks, Physica B 130, 6 (1985).

${ }^{17}$ J. Trygg et al., Phys. Rev. Lett. 75, 2871 (1995).

${ }^{18}$ G.Y. Guo, Phys. Rev. B 55, 11619 (1997).

${ }^{19}$ B.T. Thole et al., Phys. Rev. Lett. 68, 1943 (1992).

${ }^{20}$ P. Carra et al., Phys. Rev. Lett. 70, 694 (1993).

${ }^{21}$ G.Y. Guo et al., J. Magn. Magn. Mater. 148, 66 (1995).

${ }^{22} T_{z}=\left\langle\beta \hat{T}_{z}\right\rangle$ with $\hat{T}_{z}=\frac{1}{2}[\sigma-3 \hat{r}(\hat{r} \cdot \sigma)]_{z}$ where $\beta$ and $\sigma$ are the Dirac and Pauli matrices, respectively. 